Prostata-Phosphatase charakteristische Erscheinung (7, 17). Das Auftreten von vier Aktivitätszonen steht in Übereinstimmung mit den Ergebnissen der immunologischen Untersuchung von SHULMaN und FerBer (7), die durch Doppeldiffusion vier phosphatasespezifische Präzipitationslinien erhielten. Allerdings bringt die Anwendung der Disk-Elektrophorese für den qualitativen Nachweis der Phosphatase-Isoenzyme den Vorteil eines wesentlich geringeren zeitlichen und experimentellen Aufwandes mit sich, da z. B. langwierige Immunisierungen von Versuchstieren überflüssig sind.

Bei einer Elektrophorese wurde zurPrüfung des Temperatureinflusses die Kühlung abgeschaltet und die Trennung bei Zimmertemperatur vorgenommen. Unter diesen Bedingungen erreicht die Temperatur im Trenngel etwa $40^{\circ}$ (12). Dabei ist bereits eine beträchtliche Inaktivierung des Enzyms zu erwarten (1, 2, 8, 9). Das Ergebnis dieses Versuches erwies auch, daß die Aktivität der sauren Phosphatase fast völlig verschwunden war. Nach einer Inkubationszeit von 90 Min. war nur eine schwache Zone zu erkennen, diesmal auf der Höhe der Protein- bande Nr. 1. Auch das Proteinmuster (Abb. 2c) erschien gegenüber der Auftrennung bei $4^{\circ}$ wesentlich diffuser, wie der Vergleich der Abb. 2a mit 2c zeigt. Dies unterstreicht die Notwendigkeit einer wirksamen Kïblung. Das einfache Einbringen der gesamten Apparatur in einen Kühlraum und Absenken der Stromstärke (12) erscheint wenig befriedigend, da hierbei einerseits eine lokale Utberhitzung im Trenngel nicht ausgeschlossen werden kann und andererseits die Laufzeit verlängert wird. Auch beim vollständigen Eintauchen der Trennröhrchen in den unteren Elektrolyten (18) wird die Kühlung der Trennröhrchen nur durch Konvektion bewirkt und erfordert weiterhin die Außenkühlung des gesamten Apparates. Demgegenüber ist bei der beschriebenen Apparatur durch den direkten Kontakt des strömenden Kühlmediums mit den Trennröhrchen eine rasche und vollständige Abführung der Stromwärme gewährleistet, ohne daß im Kühlraum gearbeitet oder die Stromstärke erniedrigt werden muß.

Herm H. Kınx danke ich für die Anfertigung der ElektrophoreseApparatur.

\title{
Literatur
}

1. Vernon, C. A., J. Gauldie, J. M. Hanson, J. M. Humphreys, P. E. SMrth, A. J. Lawrence und B. E. C. Banks, Nature London 208, 382 (1965). - 2. Ostrowsky, W. und A. Tsugrta, Arch. Biochem. Biophysics 94, 68 (1961). - 3. Ostrowsky, W. und J. Rybarska, Biochim. biophysica Acta (Amsterdam) 105, 196 (1965). -4. Angelettr, P., B. Moore, V. Suntzeff und R. Gayle, Proc. Soc. exp. Biol. Med. 108, 53 (1961). - 5. SuR, B. K., D. W. Moss und E. J. King, Biochem. J. 84, 55 P (1962). - 6. BeCKMaN, L., Isoenzyme Variations in Man-Monographs in Human Genetics, Bd. 1 S. 36f. S. Karger AG. Basel-New York (1966). - 7. SHULnan, S. und J. M. Ferber, J. Reprod. Fert. 11, 295 (1966). - 8. JACoBbson, K., Scand. J. Clin. Laborat. Invest. 12, 367 (1960). -
9. Woodard, H. G., Amer. J. Med. 27, 902 (1959). - 10. LonDon, M. und P. B. Hudson, Arch. Biochem. Biophysics 46, 141 (1953). - 11. Richterich, R., Klinische Chemie, S. 360. Akad. Verlagsges. Frankfurt/M. (1965). - 12. Davis, B. J., Ann. N. Y. Acad. Sci. 121, 404 (1964). - 13. Rogers, L. J., Biochim. biophysica Acta (Amsterdam) 94, 324 (1965). - 14. Maurer, H. R., diese Z. 4, 85 (1966). - 15. Williams, D. E. und R. A. Reisfeld, Ann. N. Y. Acad. Sci. 121, 373 (1964). - 16. Allen, J. M. und J. Gockerman, Ann. N. Y. Acad. Sci. 121, 616 (1964). - 17. ABUL-FADL, M. A. M. und E. J. Krng, Biochem. J. 42, XXVIII (1942). - 18. Clark, J. T., Ann. N. Y. Acad. Sci. 121, 428 (1964).

\section{Kinetische Messung der $\alpha$-Amylase-Aktivität durch amyloklastische Endpunktbestimmung auf Papier}

\author{
Von G. Hillmann, G. Beyer und R. Steger \\ Aus dem Chemischen Institut der Städtischen Krankenanstalten Nürnberg (Vorstand: Prọf. Dr. G. Hillmann) \\ (Eingegangen am 22. Dezember 1966)
}

\begin{abstract}
Die amyloklastische Endpunkt-Bestimmung der $\alpha$-Amylase-Aktivität wird durch kontinuierliches Auftropfen von $\alpha$-Amylose/AmylaseMischungen, die Jodid- und Jodat-Ionen enthalten, auf angesäuertes Papier bei Raumtemperatur durchgeführt. Die Zeit vom Zusammengeben der Enzym-Substrat-Mischung bis zum auf dem Papier leicht sichtbaren Farbwechsel von Blau (Jod/Stärke) nach Gelbbraun (Jod) wird registriert. $\alpha$-Amylase-Einheiten werden aus SomoGyr's Faktor/Zeit-Beziehung abgeleitet.
\end{abstract}

The amyloçastic endpoint-determination of $\alpha$-amylase-activity is performed by the continuous dropping of buffered $\alpha$-amylase-amylosemixtures, containing $\mathrm{J}^{-}$and $\mathrm{JO}_{13}{ }^{-}$-anions, on to acidified paper at room-temperature. Time is registered from the initiation of the enzymesubstrate-mixture to an easily visable colour-change (jodine-starch-blue to jodine-brown-yellow), produced sharply on the paper. $\alpha$-amylase units are derived from Somogyr's factor-time-relation.

Die meisten modernen Routineverfahren zur Bestimmung der $\alpha$-Amylaseaktivität in Körperflüssigkeiten beruhen auf der diskontinuierlichen photometrischen Messung der Extinktionsabnahme des Jodstärkekomplexes (1). Die Rationalisierungsmöglichkeiten für Serienmessungen sind durch den großen physiologischen Variationsbereich, der häufig mehrfache Probenver- dünnungen erforderlich macht, sowie durch die verschiedenen Reagenzienpipettierungen begrenzt. Die Präzision der amyloklastischen Endpunktmethode (2) (Zeitmessung bis zum Eintritt des Farbumschlags von blau nach hellbraun) wird durch die Schwierigkeit in der Festlegung des Farbumschlags beeinträchtigt. Diese im Prinzip einfachste kinetische Messung ist wegen der 
häufigen Probeentnahme für die Jodstärkereaktion für Serienuntersuchungen wenig geeignet.

Eine Rationalisierung und Erhöhung der Präzision der kinetisch-amyloklastischen Methode ergibt sich nach unseren Untersuchungen aus zwei Modifikationen:

1. Die Amylasereaktion wird bei Zimmertemperatur (22-240) durchgeführt. Die für $\alpha$-Amylase-Aktivitätsbestimmungen in der Literatur angegebene Inkubationstemperatur von $37^{\circ}$ bzw. $40^{\circ}$ ist konventionell und reaktionskinetisch nicht erforderlich.

2. Die Glucosidspaltung wird in Gegenwart von Jodidund Jodat-Ionen durchgeführt. Im gepufferten Reaktionsansatz wird die Amylaseaktivität nach eigenen Untersuchungen durch Jodid- und Jodat-Ionen nicht beeinflußt.

Diese Modifikationen ermöglichen eine einfache kinetische Meßtechnik ohne Photometer.

Als Substrat wird Amylose verwendet. Die für alle amyloklastischen Verfahren grundlegende Jodstärkereaktion beruht auf der Bildung von blauen Jodeinschlußverbindungen mit Amylose (3). Wegen des stark wechselnden Amylosegehaltes von Stärkepräparaten erscheint die für einen Vergleich von Amylasebestimmungen in verschiedenen Laboratorien notwendige Substratstandardisierung nur durch Verwendung von Amylose erreichbar. Der einzige methodische Nachteil bestand bisher in der schlechten Löslichkeit der kommerziell zugänglichen Amylosepräparate sowie einer unkontrollierbaren Retrogradation in Pufferlösungen. Mit dem standardisierten Amylosepräparat der Fa. Merck („Amylase Merckotest") steht ein brauchbares Substrat zur Verfügung. Ein für die direkte Messung der Glucosidspaltung geeignetes synthetisches Substrat der $\alpha$-Amylase existiert bisher nicht.

Durch kontinuierliches Auftropfen des Enzym-AmyloseAnsatzes bei Zimmertemperatur auf säuregetränktes Papier ergibt sich eine auf weißem Untergrund gut sichtbare Jodstärkereaktion in statu nascendi. Die den Reaktionsverlauf darstellende Abnahme der Blaufärbung der Farbflecken über eine Violett-dunkelbraun-Färbung bis zur Gelbbraunfärbung des Jodleerwertes entspricht dem Gehalt an jodbindenden Polysacchariden. Der Farbumschlag ist auf Papier leichter zu verfolgen als in Lösung und einer manuellen Titration mit Farbindikatoren bezüglich der Präzision vergleichbar. Die Zeit vom Start der Enzymsubstratmischung bis zum Farbumschlag nach gelbbraun wird in Min. bzw. in Sek. gemessen. Diese Methodik ergibt bei Einhaltung der Standạtdbedingungen eine enzymkinetisch korrekte Bestimmung der $\alpha$-Amylaseaktivität, da die Proportionalität $z$ wischen Enzymkonzentration und reziproker Zeit vom Verlauf der Zeitumsatzkurve unabhängig ist. Die üblichen Amylaseeinheiten (STREET/ Close, SOMogyr, Int. Einh.) werden nach dem durch Jodbindungsmessung oder Reduktionsverfahren ermittelten Substratumsatz in einer Zeiteinheit definiert, unter der Annahme einer Proportionalität zwischen Enzymkonzentration, Zeit und Umsatz (Reaktion 0.
Ordnung). Die Reaktion der $\alpha$-Amylase mit hochpolymeren Polysacchariden stellt jedoch keine Reaktion 0. Ordnung dar, da stets Spaltprodukte mit unterschiedlicher Enzymaffinität auftreten. Die auf den Abbau von Stärkemengen bezogenen Amylaseeinheiten stellen somit Annäherungswerte dar.

Die Umrechnung der gemessenen Zeitwerte in die bekannten Amylaseeinheiten erfolgt nach der von Somogyr angegebenen Formel: Faktor/Zeit = Amylaseeinheiten/ $100 \mathrm{ml}$ (2). Die Faktorermittlung beruht auf parallelen Amylaseaktivitätsbestimmungen von Serum- und Harnproben nach der angegebenen kinetischen Methode sowie nach Street/Close (3), da nur die Amylaseeinheiten nach STREET/Crose auf einen Amyloseumsatz (bei $37^{\circ}$ ) bezogen werden. Die bei Zimmertemperatur gemessene Zeit bis zum jodometrisch bestimmten Substratumsatz unter Standardbedingungen wird durch den von uns empirisch ermittelten Faktor 1200 in Amylaseeinheiten nach Street/Close bei $37^{\circ}$ umgerechnet. StreET/ Close-Einheiten und Somogyr-Einheiten sind nach den Literaturangaben durch den Faktor 3,1 transponierbar (4). (Somogyr-Einheiten werden auf den Abbau von Stärkepräparaten bezogen. Wegen der bekannten Schwankungen im Amylose-Amylopektin-Verhältnis in Stärkepräparationen ist die Verwendung des rechnerisch einfacheren Faktors 3 u. E. vertretbar.)

Eine Faktorberechnung für Internationale Einheiten wird nicht angegeben, da über die Umrechnung von Somogri-Einheiten in Internationale Einheiten keine einheitliche Auffassung in der Literatur besteht (5) und eine direkte Messung des Substratumsatzes in Mikromol/Min./laus methodischen Gründen nicht möglich ist. Der Substratumsatz kann durch Jodometrie nur indirekt, durch Reduktionsverfahren wegen der geringen Empfindlichkeit in der Initialphase (6) nur annähernd gemessen werden. Eine exakte Richtigkeitskontrolle von $\alpha$-Amylaseaktivitätsbestimmungen ist aus den obengenannten Gründen z. Z. nicht möglich. Eine Umrechnung von Zeitwerten in WoHLGEMUTH-Einheiten nach einer Eichkurve ist zwar möglich, aus reaktionskinetischen Gründen jedoch nicht angezeigt.

\section{Methodik}

Reagenzien

Substrat-Puffer-Lösung, $1 l$ enthält: 0,2 g Amylose (Fa. Merck); $0,015 \mathrm{M} \mathrm{NaCl} ; 0,02 \mathrm{M}$ Phosphatpuffer $\mathrm{pH} 7,1 ; 0,007 \mathrm{M} \mathrm{KJ} ; 0,0007 \mathrm{M}$ $\mathrm{KJO}_{3}$. (Die Herstellung dieser Lösung kann in einfacher Weise durch Verwendung des Substrat-Puffer-Gemisches der Merckotestpackung $\alpha$-Amylase erfolgen, wobei die der Testpackung beigefügte Jodid-Jodat-Lösung dem Puffer-Substrat-Gemisch zugefügt wird, (Gesamtrolumen 1l). Die Haltbarkeit der Lösung ist von der Vermeidung mikrobieller Infektion abbängig. Durchschnittliche Haltbarkeit ohne Einhaltung streng steriler Kautelen im Eisschrank etwa 14 Tage. Haltbarkeitskontrolle durch jodometrische Messung der Amylosekonzentration.

Bei Verwendung der automatischen Mikromethode werden auf $1 \mathrm{l}$ Substrat-Puffer-Gemisch $10 \mathrm{~g}$ Dextran 2000 (Fa. Pharmacia) zugesetzt. Durch den Zusatz von Dextran 2000 wird der Amyloseabbau nicht beeinflußt. Die Abtropfgeschwindigkeit der EnzymSubstratpufferlösung aus den Glasspritzen wird durch die erhöhte Viskosität erniedrigt. Hierdurch entstehen perlartig aneinander gereihte Farbflecke. Die Abmessung des Umschlagpunktes wird damit erleichtert. 
Saugfähiges, dickes Clbromatograpljiepapier (Chromatographiepapier Schleicher \& Schüll Nr. 2668 bzw. Macherey \& Nagel Nr. 866), getränkt mit $1 \mathrm{M}$ Citronensäurelösung bzw. $1 \mathrm{M} \mathrm{KHSO}{ }_{4}$-Lösung. a-Amylase-Kontrollseren (nach Faktorrechnung geeicht).

\section{Durchführung der Messung}

\section{Automatisierte Metbode}

$0,1 \mathrm{~m} l$ Harn bzw. $0,5 \mathrm{~m} l$ Serum werden mit $4 \mathrm{~m} l$ Substrat-Puffer-Lösung (mit Dextran 2000) gemischt und sofort in Rekordspritzen (Ganzglasspritzen $5 \mathrm{ml}$ mit 20er Kanüle) eingefüllt. Die gleichmäßig austretenden Tropfen werden durch das mit automatischem Vorschub kontinuierlich transportierte säuregetränkte Papier $(35 \times 40 \mathrm{~cm})$ absorbiert.

Durch die Jodstärkereaktion in statu nascendi werden an der Auftropfstelle blaue Farbflecke gebildet. Entsprechend dem Amylosegehalt geht die Blaufärbung über eine Dunkelbraunfärbung bis zum Endpunkt der Jodleerfärbung über. Die Zeitmessung vom Anfang der Substrat-Enzym-Mischung bis zum Endpunkt erfolgt durch Ablesung der durch den Vorschub gegebenen Wegstrecke in $\mathrm{cm}$. Bei der nachstehend angegebenen Apparatur wird der Papiervorschub in 24 Min. durchgeführt. Durch Verwendung eines geeichten Lineals ist eine direkte Ablesung der Enzymeinheiten möglich.

\section{Beschreibung der Apparatur}

Auf der Grundplatte eines rechteckigen Gehäuses aus lackiertem Aluminiumblech $(762 / 362 / 96 \mathrm{~mm})$ ist ein Densitometer (Zusatzgerät zum Universal-Photometer "Vitatron" der Fa. Dieren, Holland) austauschbar angeschraubt. Die Transportbänder des Densitometers bewegen eine abnehmbare Schubplatte aus filzbelegtem Plexiglas $(410 / 352 / 10 \mathrm{~mm}$ ) mit konstanter (regulierbarer) Geschwindigkeit. Die Schubplatte wird seitlich durch je 6 rostfreie Kugellager geführt. 2 planparallele Plexiglasleisten $(390 / 30 / 10 \mathrm{~mm}$ ) mit je 12 Bohrungen zur Aufnahme der Glas-Rekordspritzen $(5 \mathrm{~m} l)$ sind auf seitlichen Halteblechen quer zum Transportband angeschraubt. Das Transportband ist auf den Durchlauf von $400 \mathrm{~mm}$ Schublänge in $24 \mathrm{Min}$. eingestellt. Die säuregetränkten, abgepreßten Chromatographiepapierbögen $(35 \times 40 \mathrm{~cm})$ werden auf die glatte Oberfläche der Schubplatte aufgelegt. Nach beendetem Durchlauf schaltet das Transportband durch Kontaktschaltung der Schubplatte automatisch ab unter gleichzeitiger Auslösung eines Signals.

$$
\begin{aligned}
& \text { Berechnung der } \alpha \text {-Amylaseaktivität in } 0,1 \mathrm{ml} \text { Probe } \\
& \frac{1200}{\text { gemessene Zeit in Min. }}=\text { STREET/CLOSE-Einheiten } / 100 \mathrm{ml} \\
& \frac{3600}{\text { gemessene Zeit in Min. }}=\text { SOMOGYI-Einheiten } / 100 \mathrm{ml}
\end{aligned}
$$

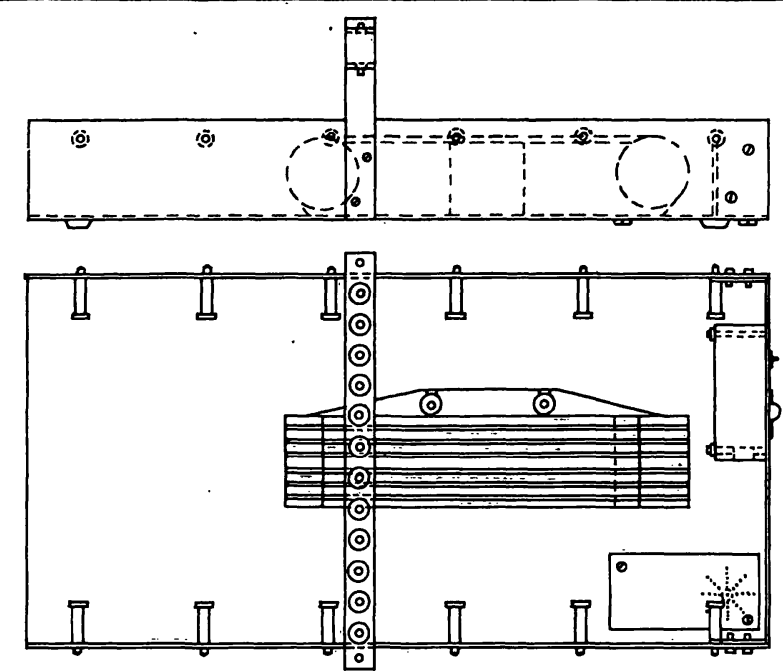

Abb. 1. Skizze des Amylase-Meßgerätes

Lịeferbar durch Fa. Vitatron, Dieren, Holland

Präzision

Blindversuch in der Serie $(\mathrm{N}=20), \overline{\mathrm{X}}=12,0$ Min.

Standardabweichung $(\mathrm{s})=0,6 \mathrm{Min}$.

Variationskoeffizient $(\mathrm{VK})=5 \%$

Empfindlichkeit (2s) $=1,2$ Min.

\section{Schnellmetbode (Screening-Test).}

$1 \mathrm{~m} l$ Harn (Serum) wird in einer Rekord-Spritze mit 2er Kanüle (bzw. Reagenzglas oder Flasche mit Tropfaufsatz) mit $4 \mathrm{~m} l$ Substrat-Puffer-Lösung rasch vermischt und unmittelbar danach eine Stoppuhr in Gang gesetzt. Der Enzym-Substrat-Ansatz wird kontinuierlich auf das säuregetränkte Papier getropft. Nach dem Umschlag der Farbe nach gelbbraun wird die Uhr gestoppt. Wenn der Umschlagspunkt unter 15 Sek. liegt, wird der Reaktionsansatz mit $0,1 \mathrm{ml}$ Harn (Serum) wiederholt. Ikterische Seren können nicht verwandt werden. Eine Lipämie stört nicht. Die Zeitmessung erfolgt bis 180 Sek. (Orale Pipettierungen sind unzulässig; die Verwendung von Marburgpipetten und Zipetten ist besonders zweckmäßig.)

Berecbnung der $\alpha$-Amylaseaktivität in $1 \mathrm{ml}$ Probe

$$
\begin{aligned}
& \frac{7200}{\text { gemessene Zeit in Sek. }}=\text { STREET } / \text { CLOSE-Einheiten } / 100 \mathrm{ml} \\
& 21600
\end{aligned}
$$$$
\overline{\text { gemessene Zeit in Sek. }}=\text { SoMOGYI-Einheiten } / 100 \mathrm{ml}
$$

Ein Zeitwert von 160 Sek. entspricht etwa 40 WoHLGEMUTH-Einheiten, ein Zeitwert von 15 Sek. etwa 200 WoHLGEMUTH-Einheiten.

\section{Präzision}

Blindversuch in der Serie $(N=20), \bar{X}=107$ Sek.

Standardabweichung $(\mathrm{s})=6$ Sek.

Variationskoeffizient $(\mathrm{VK})=5,6 \%$

Empfindlichkeit $(2 \mathrm{~s})=12 \cdot \mathrm{Sek}$.

\section{Literatur}

1. Smith, B. W. und J. H. RoE, J. biol. Chemistry 179, 53(1949). Caraway, W. T., Amer. J. Clin. Path. 32, 97 (1959). Richterich, R. und J. P. Colombo, Ärztl. Laborat. 8,33 (1962). MüLLER, G., diese Z. 2, 181 (1964). - 2. Sомоgyr, M., J. biol. Chemistry 125, 399 (1938); Clin. Chem. (New York) 6, 23 (1960). - 3. StreEt, H. V. und J. R. Close, Clin. chim. Acta, (Amsterdam) 1, 256
(1956); 3, 476 (1958). - 4. Heiger, R. u. H. LANG, Ärztl. Laborat. 11, 120 (1965). - 5. RIChrerich, R. und J. P. Colombo, Ärztl. Laborat. 8, 33 (1962). Ḣelger, R. und H. LaNG, Arztl. Laborat. 11, 120 (1965). - 6. Krng, E. J., J. Clin. Path., London 16, 391 (1963).

Professor Dr.-Ing. G. Hillmann, 85 Nürnberg, Flurstr. 17 